\title{
Photometric analysis of absorbable barbed suture for periareolar closure in mastopexy
}

\author{
Allen D. Rosen ${ }^{1,3}$, Alanna M. Guzman ${ }^{2}$, Teresa Hartman ${ }^{3}$ \\ ${ }^{1}$ Department of Plastic Surgery, University Hospital, Newark, NJ 07103, USA. \\ ${ }^{2}$ Department of General Surgery, University of Puerto Rico, San Juan, PR 00936, USA \\ ${ }^{3}$ The Plastic Surgery Group, Montclair, NJ 07042, USA.
}

Correspondence to: Dr. Allen D. Rosen, 37 North Fullerton Ave., Montclair, NJ 07042, USA. E-mail: arosen@psg1.com

How to cite this article: Rosen AD, Guzman AM, Hartman T. Photometric analysis of absorbable barbed suture for periareolar closure in mastopexy. Plast Aesthet Res 2016;3:296-301.

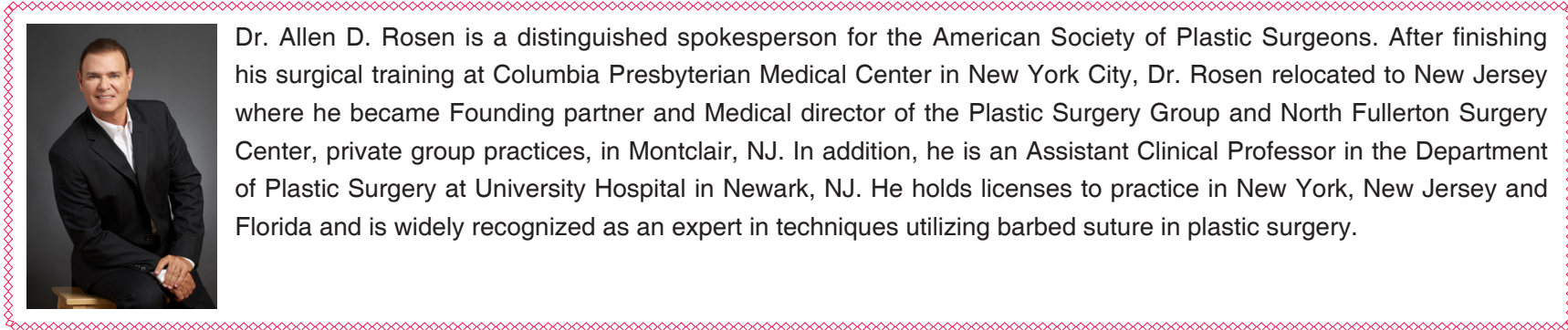

Article history:

Received: 26-05-2016

Accepted: 24-08-2016

Published: 20-09-2016

Key words:

Mastopexy,

periareolar,

absorbable barbed suture,

Quill $^{\mathrm{TM}}$ suture,

purse-string,

circumvertical,

permanent expanded

polytetrafluoroethylene suture,

GORE-TEX ${ }^{\circledR}$ suture,

breast lift

\begin{abstract}
Aim: The primary author previously described his technique for periareolar closure in mastopexy using a pinwheel interlocking purse string with absorbable barbed suture and now reports the results of a retrospective photometric analysis comparing this technique with the same closure using Gortex ${ }^{\circledR}$ suture. This study is designed to compare the degree of areolar widening and safety profile of using absorbable barbed sutures for periareolar closure versus permanent smooth suture. Methods: A retrospective chart review was conducted of all patients whose periareolar closures were performed using an interlocking purse-string technique over a 10-year period. Only patients undergoing circumvertical mastopexy were included. All had photometric evaluation and follow-up performed within 6-24 months. Results: In total, 20 patients ( 40 areolas), which were closed with absorbable barbed suture, were analyzed photometrically. In this suture group, areola size increased a mean of $4.9 \%$ from baseline, and no complications $(0 \%)$ were observed. This compared favorably with previously reported complication rates using permanent sutures and with a series of cases presented herein in which permanent smooth suture was used for purse string closure. The degree to which absorbable barbed suture controls areolar spread was shown to be significantly better than those where permanent smooth purse string techniques were employed. Conclusion: Circumvertical mastopexy closures using absorbable barbed suture was shown to be safe and effective and compared favorably to older techniques using permanent smooth suture for similar closures. This paper lends support to the safety of using absorbable barbed suture in circumareolar closures to limit areolar spread.
\end{abstract}




\section{INTRODUCTION}

According to the American Society of Plastic Surgeons, approximately 90,000 breast lifts were performed in the United States in 2013. ${ }^{[1]}$ Mastopexy closure techniques have evolved over the past decade to help limit scarring associated with the classic inverted-T technique, replacing it with Concentric (Donut) and circumvertical techniques with or without short horizontal scars. ${ }^{[2-4]}$ Even the concept of a simple purse-string periareolar closure, first described by Benelli, ${ }^{[2]}$ evolved to the more popular interlocking (pinwheel) purse-string technique, as described by Hammond et al., ${ }^{[5]}$ using permanent expanded polytetrafluoroethylene (ePTFE, GORETEX ${ }^{\circledR}$, W. L. Gore, Phoenix, AZ) suture for the deep layer. Franco et al. ${ }^{[6]}$ reaffirmed the safety and reliability of the interlocking purse-string periareolar closure using ePTFE, by evaluating a retrospective series of 50 patients who underwent augmentation mastopexy. They found a complication rate of $6 \%$ specifically associated with infected ePTFE requiring removal of this permanent foreign body. Other complications that we encountered with ePTFE in our work prior to 2008 included wound dehiscence, knot extrusion, suture palpability, fat necrosis, and areolar widening. Non-interlocking pursestring techniques using ePTFE prior to 2008 were occasionally complicated by herniation of the areola secondary to a "cerclage" effect with spread of the areola beyond the boundaries of the initial suture placement.

In 2008, with the introduction of absorbable barbed suture (Quill ${ }^{\mathrm{TM}}$ Knotless Tissue Closure Device, Surgical Specialties Corporation ${ }^{\circledR}$, Wyomissing, PA) we began using this new suture technology instead of ePTFE sutures for interlocking purse-string periareolar closure in our mastopexy, reduction mammoplasty, and augmentation mastopexy patient population. ${ }^{[7]}$ Demonstration of this

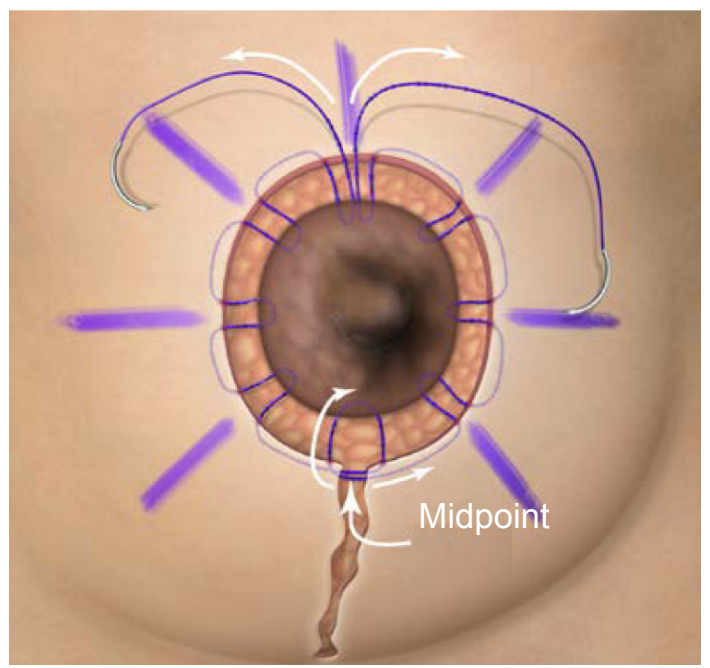

Figure 1: Interlocking purse string suture technique (deep layer with 2-0 PDO Quill) technique can be found at https://www.youtube.com/ watch? $\mathrm{v}=\mathrm{IH} \times \mathrm{KsC} 4 \mathrm{~S} 85 \mathrm{c}$. We observed a satisfactory preservation of areolar size post op and had no knotrelated infections. No herniated or distorted areolas were noted and no palpability or visibility of the suture was encountered. In addition, the long-term potential nidus for infection was eliminated. Previous studies have confirmed the cost effectiveness, safety and efficacy of using barbed suture. ${ }^{[8,9]}$

We published our work with absorbable bidirectional barbed suture for wound closure in abdominoplasty and body contouring procedures. ${ }^{[10,11]}$ Shortly after, we expanded its application to include our mastopexy closures. We undertook a formal chart review to test our hypothesis that the absorbable barbed suture, when applied using an interlocking purse-string technique, was effective in limiting the spread of the areola size post mastopexy. Swanson ${ }^{[12]}$ found photometric analysis of outcomes to be effective in assessing outcomes of breast surgery and we decided to apply similar assessment tools to our study population.

\section{METHODS}

We conducted a 10-year retrospective chart review of consecutive patients (71 patients/142 breasts) who underwent mastopexy, either alone or in conjunction with other aesthetic breast and body procedures. From 2003 to 2008 , all mastopexy closures (30 patients/60 breasts) at our center were performed using permanent ePTFE sutures for the subdermal layer and smooth absorbable Monocryl suture for deep dermal and subcuticular closure. From 2008 to 2013, mastopexy closures (41 patients/82 breasts) were performed exclusively with absorbable barbed suture. We used bidirectional PDO ${ }^{\text {TM }}$ (polydioxanone) for the deep layer and Monoderm ${ }^{\mathrm{TM}}$

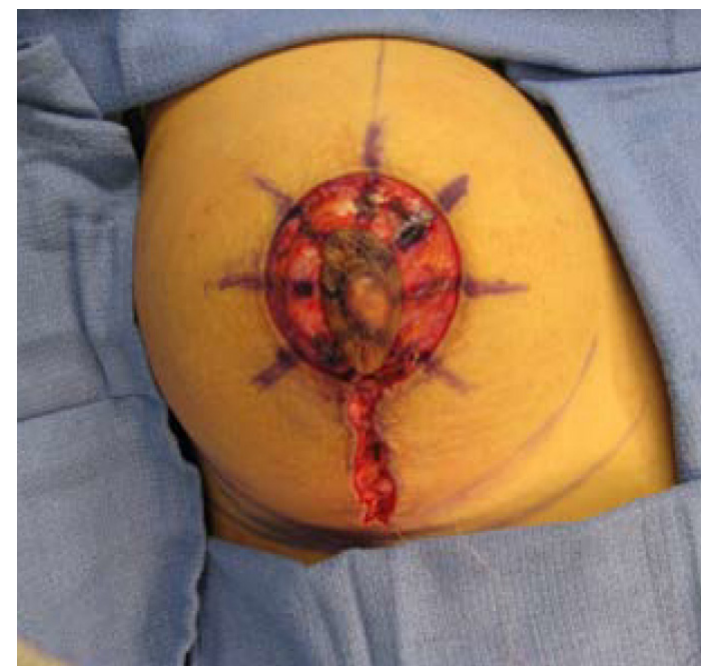

Figure 2: The periareolar wound prior to suture deployment is marked in divided quadrants 


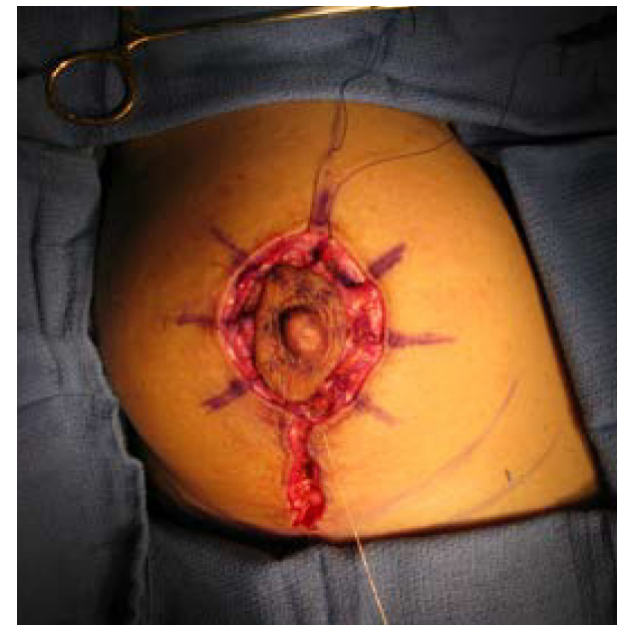

Figure 3: Intra operative photo with interlocking purse-string barbed suture prior to cinching (outlined diagrammatically in Figure 2 above).

(PGA-PCL) for subcuticular layer. Monoderm ${ }^{\mathrm{TM}}$ retains $62 \%$ of its original tensile strength at 7 days, and $27 \%$ at 14 days, with absorption essentially complete within 90 120 days, while the longer-term $\mathrm{PDO}^{\mathrm{TM}}$ retains $50-80 \%$ of its original tensile strength at 4 weeks, with absorption essentially complete within 180 days. ${ }^{[13,14]}$

We selected a patient population specifically to limit variables related to skin tension forces. We, therefore, excluded reduction mammoplasty and augmentation mastopexy and focused solely on patients undergoing mastopexy alone. The study population was further limited to patients with postoperative photographs between 6 months and 24 months after the mastopexy to minimize variables associated with aging.

The final analysis was performed on 20 eligible patients (40 breasts) in whom closures were performed using absorbable barbed suture exclusively. Since no previous reports of areolar spread rates have been published, for comparison purposes, we assessed 12 eligible patients (24 breasts) in whom mastopexy closures were performed with ePTFE/Monocryl. The primary author performed all surgeries using the same circumvertical technique ${ }^{[4]}$ at the same surgical center. Diagrammatic representation of interlocking purse string technique is demonstrated in [Figure 1]. Intraoperative photos are shown below depicting the periareolar wound, prior to suture deployment [Figure 2], after suture placement [Figure 3], and after final closure [Figure 4].

In the permanent suture group, a ligature was secured with a surgeons knot at the T-zone. Cinching of the suture was performed to the desired areolar size in both groups. Data were compiled for patient demographics [age, body mass index (BMI)], medical history (hypertension, smoking status, diabetes, previous breast surgery), surgical record (technique used, additional procedures)

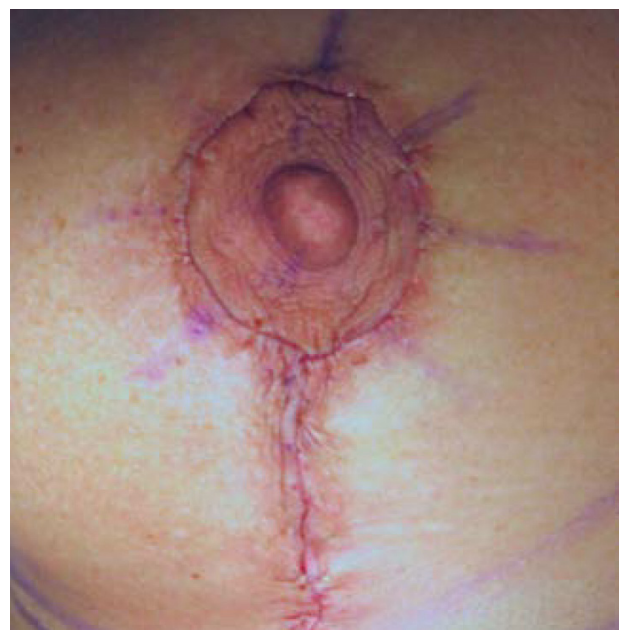

Figure 4: The final intra operative appearance of the 2-layer absorbable barbed suture closure as described

and complications. Both pre- and postoperative photos were taken with Mirror Image Software (Canfield Scientific Corp, Fairfield, NJ). Surgical areola marker size was obtained from operative report review. Followup assessments were based on photos taken between 6 and 24 months postoperatively, and postoperative areola sizes were measured using free digital photo software (GNU Image Manipulation Program/www.gimp.org). Areolar width and height measurements were completed using the GIMP software.

The primary outcome was the change from baseline areolar template size used and the photometrically measured postoperative areola size. T-test statistics were used to compare within group pre-operative areola size with postoperative areola size. Analysis of variance (ANOVA) was used to compare change from baseline scores between groups, using XLSTAT software, Version 2014.5.01. This later test was considered to be the preferred test to assess changes from baseline in studies with a non-randomized design. ${ }^{[15]}$ The incidence of complications was considered a secondary outcome.

\section{RESULTS}

The 32 patients assessed had a mean age of 41.6 years, and mean BMl of $23.5 \mathrm{~kg} / \mathrm{m}^{2}$. Patients whose mastopexy incisions were closed with absorbable barbed sutures were similar in both age and mean BMI to those closed with permanent sutures [Table 1]. The two groups were also similar in the incidence of hypertension, diabetes and in the percentage that were current smokers. Many $(12 / 32,37.5 \%)$ had a history of other relevant medical conditions. More subjects in the barbed suture closure group had undergone previous breast surgeries: $6 / 20$, $30.0 \%$ vs. $2 / 12,16.7 \%$ in the ePTFE group.

In $62.5 \%$ of cases (20/32), mastopexy was performed 
Table 1: Baseline characteristics and medical history

\begin{tabular}{lcc}
\hline Characteristic/history & Absorbable barbed suture $(\boldsymbol{n}=\mathbf{2 0})$ & Permanent suture $(\boldsymbol{n}=\mathbf{1 2})$ \\
\hline Age, years, mean (range) & $42.6(24-66)$ & $40.1(20-71)$ \\
$\mathrm{BMI}, \mathrm{kg} / \mathrm{m}^{2}$, mean (range) & $22.9(18.5-28.5)$ & $24.4(19.0-34.7)$ \\
Hypertension, $n(\%)$ & $2(10.0)$ & $1(8.3)$ \\
Current smoker, $n(\%)$ & $4(20.0)$ & $3(25.0)$ \\
Diabetes, $n(\%)$ & $1(5.0)$ & $1(8.3)$ \\
Any other relevant medical history, $n(\%)^{*}$ & $6(30.0)$ & $6(50.0)$ \\
Previous breast surgery, $n(\%)^{\dagger}$ & $6(30.0)$ & $2(16.7)$ \\
\hline
\end{tabular}

*Patients in the barbed suture group also had other relevant medical history that included a history of: leukopenia (1); kidney disease (1); rheumatoid arthritis (1); heavy scars (2); lupus and fibromyalgia (1); and high blood pressure, steroid use, blood clots, and Hodgkin Lymphoma (1); patients in the smooth suture group also had a history of: allergies and exercise-induced asthma (1); hypercholesterolemia, cough and asthma (1); breast cancer (1); cough and in-utero fibroids (1); medullary sponge kidney (1); and heart and gastrointestinal disease (1).†Patients in the barbed suture group had previously undergone bilateral mastopexy (1), breast reduction (3), lumpectomy (1) and biopsy (1); patients in the smooth suture group had previously undergone lumpectomy and axillary node dissection (1) and excision of a benign cyst

\section{Table 2: Surgical record}

\begin{tabular}{lcc}
\hline Surgical record & Absorbable barbed suture $(\boldsymbol{n = 2 0 )}$ & $\begin{array}{c}\text { Permanent suture } \\
(\boldsymbol{n}=\mathbf{1 2})\end{array}$ \\
\hline Mastopexy only, $n(\%)$ & $9(45.0)$ & $3(25.0)$ \\
Mastopexy in conjunction with other aesthetic procedures, $n(\%)^{*}$ & $11(55.0)$ & $9(75.0)$ \\
$\quad$ Abdominoplasty & $6(30.0)$ & $5(41.7)$ \\
Blepharoplasty & $3(15.0)$ & $1(8.3)$ \\
Correction of inverted nipple, bilateral $_{\text {Hernia repair }}$ & $1(5.0)$ & $0(0)$ \\
$\quad$ Liposuction $^{\dagger}$ & $2(10.0)$ & $0(0)$ \\
$\quad$ Filler to lips and/or nasolabial folds & $2(20)$ & $1(10)$ \\
$\quad$ Upper abdominal lift & $2(20)$ & $0(0)$ \\
Mastopexy technique, $n(\%)$ & $0(0)$ & $1(8.3)$ \\
$\quad$ Circumvertical & $20(100)$ & $12(100)$ \\
\hline
\end{tabular}

*Some patients had multiple additional procedures. ${ }^{\dagger}$ Patients in the barbed suture group had power-assisted lipoplasty of the hips, abdomen and thighs (1) and bilateral anterior axillary liposuction (1). The patient in the smooth suture group had liposuction of the neck

in conjunction with various other aesthetic surgical procedures [Table 2]. Of these, abdominoplasty was the most common procedure; it was performed in $30 \%$ of procedures in which the mastopexy incision was closed with absorbable barbed suture, and in $41.7 \%$ of procedures in which ePTFE sutures were used.

Follow-up photos used for the assessment of areola size were taken a mean of 11.2 months postoperatively. Mean follow-up times were similar for both cohorts: 10.7 and 12.0 months, respectively [Table 3]. Among patients in the ePTFE suture group, areola size for both breasts increased a mean of $0.49 \pm 0.57 \mathrm{~cm}$ from a baseline mean of $3.90 \pm 0.18 \mathrm{~cm}(P=0.011)$, representing a $12.5 \%$ increase from baseline.

Using absorbable barbed suture, areola size increased a mean of $0.20 \pm 0.70 \mathrm{~cm}$ from a baseline mean of 4.11 $\pm 0.24 \mathrm{~cm}(P=0.236)$, representing a $4.9 \%$ increase from baseline. The increase in areola size was a mean of $0.29 \pm 0.16 \mathrm{~cm}$ less for patients in the absorbable barbed suture group; however, the difference between groups in the change from baseline areola size did not reach statistical significance ( $P=0.092$, based on ANOVA).
No major or minor complications were reported for any patient who underwent mastopexy closure using the absorbable barbed suture [Table 4]. In contrast, 2 of the 24 breasts $(8.3 \%)$ where ePTFE suture was used had complications. In one case, complications included wound dehiscence and infection; this patient had a history of breast cancer. In a second case, complications were limited to wound dehiscence and fat necrosis; this patient had a history of hypertension and diabetes.

\section{DISCUSSION}

We believe the unique property of the barbed suture allows redistribution of tension forces throughout the entire length of the closure, and is particularly effective for round closures under circumferential tension. Smooth sutures, whether permanent or absorbable, allow tissues to slide along the length of the suture and "bunch up" similar to the way a shower curtain slides unevenly along its rod. Furthermore, we have found that long-acting absorbable sutures provide enough strength to allow quality tension-free healing to occur without leaving permanent foreign body material beneath thin areolar 
Table 3: Mastopexy closure with barbed and smooth sutures

\begin{tabular}{lcc}
\hline Surgical record & $\begin{array}{c}\text { Absorbable barbed suture } \\
(\boldsymbol{n}=\mathbf{2 0})\end{array}$ & Permanent suture $(\boldsymbol{n}=\mathbf{1 2})$ \\
\hline Mastopexy only, $n(\%)$ & $9(45.0)$ & $3(25.0)$ \\
Mastopexy in conjunction with other aesthetic procedures, $n(\%)^{*}$ & $11(55.0)$ & $9(75.0)$ \\
$\quad$ Abdominoplasty & $6(30.0)$ & $5(41.7)$ \\
$\quad$ Blepharoplasty & $3(15.0)$ & $0(0.3)$ \\
$\quad$ Correction of inverted nipple, bilateral & $1(5.0)$ & $0(0)$ \\
$\quad$ Hernia repair & $2(10.0)$ & $1(10)$ \\
$\quad$ Liposuction $^{\dagger}$ & $2(20)$ & $0(0)$ \\
$\quad$ Filler to lips and/or nasolabial folds & $2(20)$ & $1(8.3)$ \\
$\quad$ Upper abdominal lift & $0(0)$ & $12(100)$ \\
Mastopexy technique, $n(\%)$ & $20(100)$ & \\
$\quad$ Circumvertical & & \\
\hline
\end{tabular}

${ }^{*} t$-test for within group pre-op areola marker size vs. post-op areola size, using Excel. ${ }^{\dagger}$ Analysis of variance for between groups change from baseline scores, using XLSTAT software, Version 2014.5.01

Table 4: Postoperative complications

\begin{tabular}{lcc}
\hline Complication & Absorbable barbed suture $(\boldsymbol{n}=\mathbf{4 0})$ & Permanent suture $(\boldsymbol{n}=\mathbf{2 4})$ \\
\hline Any complication, $n(\%)$ & $0(0)$ & $2(8.3)$ \\
Wound dehiscence, infection & $0(0)$ & $1(4.15)$ \\
Wound dehiscence, fat necrosis & $0(0)$ & $1(4.15)$ \\
\hline
\end{tabular}

tissues. Absorbable suture eliminates long-term suture palpability, both at the knot and the circumferential portion of the permanent suture.

We also strongly believe that the tension relieving nature of the interlocking purse-string technique is further enhanced by the tension redistribution noted with barbed sutures. The combination of the novel interlocking technique and the improved absorbable barbed suture technology acts in synergy to reduce wound tension and minimize complications.

Various complications in procedures involving periareolar closures have been reported in the literature, most typically in association with breast reduction or augmentation/mastopexy surgeries ${ }^{[16-21]}$ Delayed wound healing, wound dehiscence, hematoma or seroma formation, wound infection, fat necrosis, stitch abscess, diminished nipple sensation and scarring have been reported but limited data are available for mastopexy alone. Franco's review ${ }^{[6]}$ using interlocking ePTFE reports similar complications. Since the wound healing problems listed in the permanent suture cohort in our review occurred in patients with systemic comorbidities, this could have played a role in the healing process. In addition, the retrospective nature of this review and the small, uneven sample size do not allow us to make statistically significant comparisons between groups, but the limited sample did show that areolar size was well preserved and no complications were seen in this particular group when closing the periareolar tissues exclusively with absorbable barbed suture.

Although complication rates for mastopexy are well reported in our literature, the effectiveness of controlling the degree of areolar spread has not. Many authors have made references to this outcome, but the incidence rates in a population of mastopexy patients without augmentation or volume reduction have not been previously reported. For this reason, we included a small sample of our own patients to serve as a comparative cohort in assessing post op areolar widening, an important parameter to demonstrate efficacy of periareolar closure techniques.

Certainly, some limitations of this study are inherent to the nonrandomized retrospective study design. A prospective, randomized multicenter trial with a larger sample size would be necessary to validate our observational findings. In addition, since only barbed suture was used we could not assess whether smooth absorbable suture might perform as well at lower cost.

Although the mean follow-up period was similar between groups, the range was wide varying from 6 to 24 months. It would have been preferable if all follow-up photographs had been recorded within an even narrower window to better restrict changes in breast size that may otherwise been due to weight gain or loss or other variables. It is possible that direct postoperative areolar measurements with calipers would have been preferable to photometric measurements, although both methods have a degree of inherent inaccuracy. We are also aware that due to the contractile properties of the nipple areolar complex, areolar size may vary based on environmental conditions, room temperature, stress and other unforeseen stimulatory factors.

In our surgical practice, the use of absorbable barbed 
suture for circumareolar closure was found to be effective in limiting post op areolar widening and was without complications. We present our data as a comparative photometric and clinical analysis for surgeons considering making the "leap of faith" and performing periareolar closure with absorbable barbed suture alone.

The tension reducing benefits of the interlocking pursestring technique combined with absorbable barbed suture technology yields predictable areolar size outcomes and minimizes suture related complications in mastopexy. As plastic surgeons continue to evolve and explore new suture technologies and techniques, this study will support the safety and efficacy of doing so exclusively with absorbable barbed suture.

\section{Financial support and sponsorship}

None.

\section{Conflicts of interest}

There are no conflicts of interest.

\section{Patient consent}

Not applicable.

\section{Ethics approval Not applicable.}

\section{REFERENCES}

1. American Society of Plastic Surgeons. 2013 Plastic Surgery Statistics Report. Available from: www.plasticsurgery.org. [Last Accessed on 24 June 2014].

2. Benelli L. A new periareolar mammoplasty: the "round block" technique. Aesthet Plast Surg 1990;14:93-100.

3. Lejour M. Vertical mammoplasty and liposuction of the breast. Plast Reconstr Surg 1994;94:100-14.

4. Hall-Findlay EJ. A simplified vertical reduction mammoplasty: shortening of the learning curve. Plast Reconstr Surg 1999;104:748-59.

5. Hammond DC, Khuthaila DK, Kim J. The interlocking Gore-Tex suture for control of areolar diameter and shape. Plast Reconstr Surg
2007;119:804-9

6. Franco J, Kelly E, Kelly M. Periareolar augmentation mastopexy with interlocking Gore-Tex suture, retrospective review of 50 consecutive patients. Arch Plast Surg 2014;41:728-33.

7. Rosen AD. Periareolar closure with barbed sutures. Aesthet Surg $J$ 2016;36:372-5.

8. Ruff G. Technique and uses for absorbable barbed sutures. Aesthet Surg $J$ 2006;26:620-8.

9. Paul MD, Budd M. Evaluating the Quill self-retaining system. Available from: http://www.plasticsurgerypractice.com/2009/03/evaluating the-quill-self-retaining-system/. [Published on 24 March 2009; Last Accessed on 08 September 2016].

10. Rosen $\mathrm{AD}$. Use of absorbable running barbed suture and progressive tension technique in abdominoplasty: a novel approach. Plast Reconstr Surg 2010;125:1024-7.

11. Rosen A, Hartman T. Repair of the midline fascial defect in abdominoplasty with long-acting barbed and smooth absorbable sutures. Aesthet Surg J 2011;31:668-73.

12. Swanson E. A retrospective photometric study of 82 published reports of mastopexy and breast reduction. Plast Reconstr Surg 2011;128:1282-301

13. Quill ${ }^{\mathrm{TM}}$ self-retaining system (SRS) comprised of Monoderm ${ }^{\mathrm{TM}}$ (PGAPCL) synthetic absorbable surgical suture material. Instructions for use. Angiotech, Reading, PA

14. Quill ${ }^{\mathrm{TM}}$ self-retaining system (SRS) comprised of dyed PDO (polydioxanone) synthetic absorbable surgical suture material Instructions for use. Angiotech, Reading, PA.

15. Van Breukelen GJ. ANOVA versus change from baseline had more power in randomized studies and more bias in nonrandomized studies. $J$ Clin Epidemiol 2006;59:920-5.

16. Shiffman MA. Mastopexy complications. In: Shiffman MA, Di Giuseppe A, editors. Body Contouring. Berlin: Springer; 2010. p.145-7.

17. Hidalgo DA, Spector JA. Mastopexy. Plast Reconstr Surg 2013;134:e642-56

18. Stevens WG, Stoker DA, Freeman ME, Quardt SM, Hirsch EM Mastopexy revisited: a review of 150 consecutive cases for complication and revision rates. Aesthet Surg J 2007;27:150-4.

19. Stevens WG, Spring M, Stoker DA, Freeman ME, Cohen R, Quardt SM, Hirsch EM. A review of 100 consecutive secondary augmentation/ mastopexies. Aesthet Surg J 2007;27:485-92.

20. Spear SL. Augmentation/mastopexy: "surgeon, beware". Plast Reconstr Surg 2006;118:S133-4

21. Spear SL, Boehmler JH 4th, Clemens MW. Augmentation/mastopexy: a 3-year review of a single surgeon's practice. Plast Reconstr Surg 2006;118:S136-47. 